\title{
A fatal outcome after unintentional overdosing of rivastigmine patches
}

\author{
Henrik Lövborg, Anna K Jönsson and Staffan Hägg
}

\section{Linköping University Post Print}

N.B.: When citing this work, cite the original article.

Original Publication:

Henrik Lövborg, Anna K Jönsson and Staffan Hägg, A fatal outcome after unintentional overdosing of rivastigmine patches, 2012, Current drug safety, (7), 1, 30-2.

http://dx.doi.org/10.2174/157488612800492717

Copyright: Bentham Science Publishers http://www.benthamscience.com/

Postprint available at: Linköping University Electronic Press http://urn.kb.se/resolve?urn=urn:nbn:se:liu:diva-80816 


\section{A fatal outcome after unintentional overdosing of rivastigmine}

\section{patches}

Authors: Henrik Lövborg*¹, Anna K Jönsson ${ }^{1}$, Staffan Hägg ${ }^{1}$.

1. Department of drug research/Clinical Pharmacology, Faculty of Health Sciences, Linköping University, Department of Clinical Pharmacology, County Council of Östergötland, S-581 85 Linköping, Sweden *=corresponding author, Henrik.Lovborg@lio.se , +46 1010343 26, fax +4613104195

Conflict of interest: No conflict of interest declared by any of the authors.

Originality: This manuscript is not under review or published in any other journal.

Running title: Fatal overdosing of rivastigmine patches

Word count: 1418 (excl abstract and references)

Number of figures: 1 


\begin{abstract}
Background: Rivastigmine is an acetylcholine esterase inhibitor used in the treatment of dementia. Patches with rivastigmine for transdermal delivery have been used to increase compliance and to reduce side effects. Case report: We describe a 87-year old male with dementia treated with multiple rivastigmine patches (Exelon 9,5 $\mathrm{mg} / 24 \mathrm{~h}$ ) who developed nausea, vomiting and renal failure with disturbed electrolytes resulting in death.. The symptoms occurred after six rivastigmine patches had concomitantly been erroneously applied by health care personnel on two consecutive days. The terminal cause of death was considered to be uremia from an acute tubular necrosis that was assessed as a result of dehydration through vomiting. The rivastigmine intoxication was assessed as having caused or contributed to the dehydrated condition. The medication error occurred at least partly due to ambiguous labelling. The clinical signs were not initially recognized as adverse effects of rivastigmine. Discussion: The presented case is a description of a rivastigmine overdose due to a medication error involving patches. This case indicates the importance of clear and unambiguous instructions to avoid administration errors with patches and to be vigilant to adverse drug reactions for early detection and correction of drug administration errors. In particular, instructions clearly indicating that only one patch should be applied at a time are important.
\end{abstract}

Key words: adverse drug reactions, medication errors, overdose, rivastigmine, transdermal drug delivery 


\section{Introduction}

A considerable proportion of all patient safety events concern medication errors [1]. Even though many of these events do not result in adverse patient outcomes, serious patient harm or even loss of life may occur. Studies have estimated that 5-12\% percent of all hospital admissions are related to problematic use of pharmaceuticals [2,3]. Among the possible types of medication errors, administration errors are often mentioned as common and often leading to patient harm $[4,5,6]$ and can occur due to errors by either patients or health care professionals. Administration errors include dosing at the wrong time, by the wrong route or with too high or too low doses that could contribute to harm. Here we describe a case of an administration error with subsequent overdose in a patient treated with the acetylcholine esterase inhibitor rivastigmine patches for dementia.

\section{Case report}

This case was reported to the Regional Pharmacovigilance Centre in Linköping, which is a collaboration between the county councils in the Southeast region of Sweden and the Medical Products Agency in Sweden. The report described an 87-year old male with dementia. The patient was treated with rivastigmine patches (Exelon 9,5 mg/24 h, q.d.), dimethicone (200 mg t.i.d.), acetylsalicylic acid (75 mg q.d.), etilefrine (5 mg t.i.d.), alprazolam (0,5 mg q.d.), escitalopram (20 mg q.d.) and mirtazapine (15 mg q.d.). One afternoon home care services personnel found the man on the floor in his home. When lifting him into his bed, the staff noticed that he had six rivastigmine patches (Exelon 9,5 mg/24 h) attached to the upper part of his body. The recommended [7], and prescribed, dose was one patch at a time that should be changed every $24 \mathrm{~h}$. Blood pressure was 114/62 and pulse $101 \mathrm{bpm}$. The staff described, however, that he was administered six patches the day before this event, and that these patches were changed to six new patches in the morning by the same person. Misinterpretation of the patient information leaflet accompanying the patch (figure 1) was the reason for this mistake according to the staff. In retrospect the staff described that the patient after the application of the first six patches suffered from nausea, vomiting and confusion but this was not put into relation with the use of the drug at that time point. All patches were removed and after consulting a physician it was decided that the man should stay in his home with more frequent visits to monitor blood pressure and pulse. In case of bradycardia a physician should be contacted.

Three hours after removing the patches the blood pressure was 150/80 $\mathrm{mmHg}$ and pulse $106 \mathrm{bpm}$. At a visit 9 hours (1 a.m.) after removing the patches the man was again found on the floor. Furnishing and decorations had been torn down and the man had scrape wounds on arms, knees and the forehead. The man was conscious but 
dizzy, the pulse was fluctuating with a frequency of $45 \mathrm{bpm}$. Blood pressure was $120 / 80 \mathrm{mmHg}$. Thereafter the man was transferred by ambulance to the emergency department of the nearby county hospital.

On admittance to the emergency he was in his usual state not showing any clinical signs of rivastigmine overdose, but serum creatinine was $149 \mu \mathrm{mol} / \mathrm{L}$ and he presented signs of urinary tract infection, a rivastigmine labelled adverse drug reaction described in the Exelon Summary Product Characteristics (SPC). During the following days he developed acute renal failure with increased potassium levels and decreased calcium. Despite that efforts were made to adjust salt and fluid balance, the condition progressively deteriorated with serum creatinine rising to $1035 \mu \mathrm{mol} / \mathrm{L}$ and urea $80 \mathrm{mmol} / \mathrm{L}$. The patient died in hospital 19 days after the overdose. The terminal cause of death was considered uremia from an acute tubular necrosis that was assessed as a result of dehydration through vomiting. The treatment with the rivastigmine patches were assessed as having caused or contributed to the dehydrated condition.

\section{Discussion}

In this case unintentional and inappropriate use of multiple patches of rivastigmine is described. The same day as the first six patches were applied the patient presented typical signs of dose-related rivastigmine adverse drug reactions such as confusion and nausea [7]. In another case [8] an overdose due to a medication error similar to the one we describe in this report is described. In that case a 80-year-old woman with dementia who presented to a hospital emergency department complaining of light-headedness, vomiting, diarrhoea and lower extremity muscle pain, all well known side effects of rivastigmine. Examination showed fasciculations and miotic pupils. On her body nine rivastigmine patches $\left(\operatorname{Exelon}^{\circledR}, 9,5 \mathrm{mg} / 24 \mathrm{~h}\right)$ were found. It was determined that the patient placed the nine patches on her body 5 days prior to this event and that the symptoms gradually increased during this period. She was discharged 3 days later in a stable condition. In two published cases of oral overdoses typical signs of cholinergic syndrome including dizziness, vomiting, salivation, miosis and cardiac and respiratory effects are described $[9,10]$. In both these cases the patients also presented with transient bradycardia, as is described in our case. Moreover, rivastigmine has in clinical trials been associated with renal failure, which was part of the symptoms presenting in the case we describe [11].

The adverse reactions presented by the patient after application of the six patches should have warranted further examination of the patient, or contact with a physician to clarify the cause of the symptoms. Increased effort to 
ascertain a high standard of knowledge in the group of healthcare and home care services personnel is important for a safe use of drugs. It is also important to increase early detection rate of adverse drug reactions that could be potentially serious.

Transdermal patches have been described as one way to increase compliance in Alzheimer patients [12]. In the case of rivastigmine, patches have been shown to provide similar efficacy and increased tolerability compared to rivastigmine capsules [13]. Furthermore, the aspect of convenience for caregivers and patients have been questioned, especially since application of rivastigmine patches to the exact same skin location should be avoided within 14 days according to the SPC instruction [14]. Since significant amounts of active substance can still be present in the patch at the intended time of change [15] the use of patches warrants extra care to avoid administration of multiple doses. The risk of medication errors of this type is likely to be especially high when administration of the patch is made by different individuals over time and at different locations on the body surface.

Mistakes with application of multiple patches have been described for patches for pain relief [16,17]. This shows that use of this administration route is prone to this specific type of error, especially since the treatment often is indicated in patients with advanced age or patients whose medication is controlled by ambulatory teams with frequent change of staff members. During the same year two additional reports with multiple rivastigmine patches were sent to the Regional Pharmacovigilance Centre in Linköping suggesting that the case described in this report is not an isolated event (unpublished data). In both these cases health care personnel observed two patches at the same time attached to the patients body when changing rivastigmin patches. In these cases no adverse drug reactions were described. The type of spontaneous adverse drug reaction reports described here indicates that such systems for pharmacovigilance monitoring can be used not only for establishing the safety of the drug itself, but also to find medication handling problems. Moreover, the described case stresses the importance of unambiguous labelling to ascertain a safe use of drugs. Following the report of this case the manufacturer was contacted by the Swedish Medical Products Agency to start a process of clarifying the information regarding dosing and application of the rivastigmine patches. This has now resulted in the decision by the European Medicines Agency to update the SPC with strict information about only using one patch at a time. This will hopefully reduce the risk for similar misinterpretations in the future. 


\section{Conclusion}

This case indicates the importance of clear instructions to patients and relatives as well as to health care personnel to avoid administration errors with patches. This includes verbal information by doctors, nurses and pharmacists as well as unambiguous information in SPC's and package information leaflets. In particular, instructions clearly indicating that only one patch should be applied at a time are important.

\section{Acknowledgement}

The authors declare no conflict of interest. The authors acknowledge the financial support from the Swedish Research Council. 


\section{References}

1 Leape LL, Bates DW, Cullen DJ, et al. Systems analysis of adverse drug events. Ade prevention study group. JAMA 1995; 274: 35-43.

Mjorndal T, Boman MD, Hagg S, et al. Adverse drug reactions as a cause for admissions to a department of internal medicine. Pharmacoepidemiol Drug Saf 2002; 11: 6572.

3 Pirmohamed M, James S, Meakin S, et al. Adverse drug reactions as cause of admission to hospital: Prospective analysis of 18820 patients. BMJ 2004; 329: 15-9. 4 Kiekkas P, Karga M, Lemonidou C, Aretha D, Karanikolas M. Medication errors in critically ill adults: A review of direct observation evidence. Am J Crit Care 2011; 20: $36-44$.

5

Mills PD, Neily J, Kinney LM, Bagian J, Weeks WB. Effective interventions and implementation strategies to reduce adverse drug events in the veterans affairs (va) system. Qual Saf Health Care 2008; 17: 37-46.

Valentin A, Capuzzo M, Guidet B, et al. Errors in administration of parenteral drugs in intensive care units: Multinational prospective study. BMJ 2009; 338: b814. 7 European medicines agency. Exelon: Summary of product charachteristics [online], revised 11/06/2010 available from url: Http://www.Ema.Europa.Eu

Zappala M. Use of pralidoxime without atropine in rivastigmine (carbamate) toxicity. Hum Exp Toxicol 2009; 28: 599-602.

9 Brvar M, Mozina M, Bunc M. Poisoning with rivastigmine. Clin Toxicol (Phila) 2005; 43: 891-2. evidence of respiratory depression. Emerg Med J 2006; 23: 82-5. 

revised 08/2010.

Potential benefits of a transdermal patch. Curr Med Res Opin 2007; 23: 2705-13. randomized, placebo-controlled study of a transdermal patch in alzheimer's disease-rivastigmine patch versus capsule. Int J Geriatr Psychiatry 2007; 22: 456-67. convenient than capsules. Prescrire Int 2009; 18: 19. the novel daily rivastigmine transdermal patch compared with twice-daily capsules in alzheimer's disease patients. Clin Pharmacol Ther 2008; 83: 106-14. Harding AM, Chandler K. Patch identification poster and education to reduce transdermal medication errors. J Pharm Pract Res 2010; 40: 332-3.

31: $278-81$. 
Figure 1

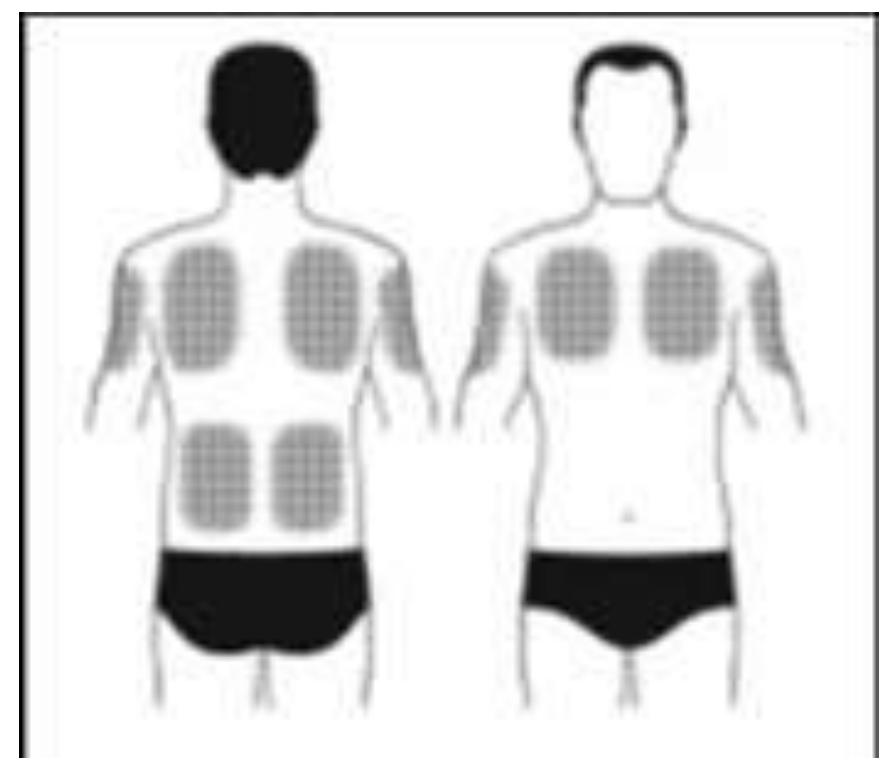

Figure legend

Illustration from Exelon patches SPC indicating suitable areas to attach a patch. 